\title{
EFEITO DE SOLUÇÕES DE ENXÁGUE NA REMOÇÃO DE RESÍDUOS DE MANCOZEB EM TOMATES DE MESA ${ }^{1}$
}

\author{
Márcia Regina Ribeiro Alves², Maria Célia Lopes Torres², Nilda de Fátima Ferreira Soares ${ }^{3}$, \\ Nathália Ramos Melo $^{3}$, Robson Maia Geraldine ${ }^{2}$, Eduardo Seiti Gomide Mizubuti ${ }^{4}$, Míriam Fontes Araujo Silveira ${ }^{2}$
}

\section{ABSTRACT \\ EFFECT OF RINSING SOLUTIONS FOR REMOVING MANCOZEB RESIDUES IN TABLE TOMATOES}

Fungicides are the pesticides most widely used for tomato cultivation, mainly dithiocarbamates. The objective of this research was to study the efficiency of dithiocarbamate mancozeb removal from table tomatoes, through rinsing processes. Analyses of tomato acidity, brix, water activity, $\mathrm{pH}$, and manganese content were carried out. After rinsing tomatoes with drinking water and solutions of vinegar (made from alcohol), sodium bicarbonate, and dihidrated sodium dichloroisocyanurate, mancozeb levels were assessed in skin and whole fruit, by quantifying the manganese content present in the samples. It was observed that the rinsing processes reduced significantly the amount of residues in the tomatoes. The reduction of mancozeb was higher in the skin, where it can be found in its highest concentration. Treatments using drinking water and sodium bicarbonate solution removed over $61 \%$ of the residues present in tomatoes skin.

KEY-WORDS: Fungicides; dithiocarbamates; Lycopersicum esculentum Mill.

\section{INTRODUÇÃO}

A cultura do tomate (Lycopersicum esculentum Mill.), seja de mesa ou para processamento industrial, é uma das mais difíceis de se produzir sem o uso de agrotóxicos, devido à alta incidência e susceptibilidade a pragas e doenças. O Brasil é classificado, pela Organização das Nações Unidas para a Agricultura e Alimentação (FAO), como um dos países onde mais se aplicam agrotóxicos de forma excessiva (Bedor et al. 2007). Os fungicidas são os mais utilizados na cultura do tomate, sendo os ditiocarbamatos os mais aplicados. Alguns metabólitos produzidos por fungici-

\section{RESUMO}

Dentre os agrotóxicos mais utilizados na cultura do tomate estão os fungicidas e, dentre estes, os mais aplicados na cultura são os ditiocarbamatos. O objetivo deste trabalho foi avaliar a eficiência dos processos de enxágue de tomates de mesa, por imersão, com água potável e soluções de vinagre de álcool, bicarbonato de sódio e dicloroisocianurato de sódio dihidratado, utilizados na remoção do fungicida mancozeb. Foram realizadas análises de acidez, brix, atividade de água $\left(\mathrm{a}_{\mathrm{w}}\right)$, $\mathrm{pH}$ e teor de manganês. Foi avaliado o teor do fungicida na película e no fruto inteiro, pela quantificação do teor de manganês presente nas amostras. Observou-se que o processo de enxágue do fruto reduz, significativamente, a quantidade de resíduos nos tomates. A redução de mancozeb foi maior na película, onde está presente em maior concentração, e os enxágues com água potável e solução com bicarbonato de sódio removeram mais de $61 \%$ dos resíduos presentes na película dos tomates.

PALAVRAS-CHAVE: Agrotóxico; ditiocarbamatos; Lycopersicum esculentum Mill.

das são prejudiciais ao homem e, por esta razão, vários estudos são realizados para avaliar a remoção deste agrotóxico (Vetorazzi et al. 1995, Hwang et al. 2002, Bastos et al. 2007). Os ditiocarbamatos são usados como fungicidas no mundo inteiro, há várias décadas, na agricultura, no cultivo de plantas ornamentais e de gramas e no tratamento do solo (Arbo et al. 2006). Tais compostos formam a mais importante classe de agrotóxicos, empregada no controle de diversas doenças fúngicas em sementes, frutos e vegetais (Arbo et al. 2006, Lima et al. 2007). Apresentam características de proteção de contato e formação de película protetora na superfície tratada, que impede a penetração

1. Trabalho recebido em ago./2008 e aceito para publicação em mar./2010 (nº registro: PAT 4593/ DOI: 10.5216/pat.v40i1.4593).

2. Universidade Federal de Goiás, Escola de Agronomia e Engenharia de Alimentos, Setor de Tecnologia de Alimentos, Goiânia,

GO, Brasil.E-mails: marcia.uft@hotmail.com, celialopes.ufg@gmail.com, robson.agro.ufg@gmail.com,miriamfas.ufg@gmail.com.

3. Universidade Federal de Viçosa, Centro de Ciências Exatas e Tecnológicas, Departamento de Tecnologia de Alimentos,

Viçosa, MG, Brasil.E-mails: nfsoares@ufv.br, nathaliarm@hotmail.com.

4. Universidade Federal de Viçosa, Centro de Ciências Agrárias, Departamento de Solos, Viçosa, MG, Brasil.

E-mail:mizubuti@ufv.br. 
de patógenos. Tem um amplo espectro e múltiplos sítios de ação. As principais formas de degradação são fotólise e hidrólise e perda durante processamento da amostra (Jardim 2007). Segundo Lemes (2005), o grupo químico dos ditiocarbamatos está presente, com maior frequência, em diversos alimentos. Em estudo realizado por Delgado \& Paumgartten (2004), o mancozeb foi o ditiocarbamato mais utilizado em propriedades rurais, apresentando percentual de $44 \%$ de presença, demonstrando o quanto o fungicida é empregado nas culturas.

Os fungicidas maneb e mancozeb contêm, em sua molécula, manganês, o qual está associado a danos no sistema nervoso central, como a síndrome que lembra o parkinsonismo (Debbarh et al. 2002, Zhou et al. 2004). Segundo Hunsche et al. (2007), o manganês corresponde a $17 \%$ do peso molecular do mancozeb. Os ditiocarbamatos são, essencialmente, determinados por métodos indiretos e os produtos da reação liberados após decomposição por ácido. Esses métodos são normalmente incapazes de distinguir entre diferentes ditiocarbamatos, uma vez que são decompostos em gás dissulfeto de carbono $\left(\mathrm{CS}_{2}\right)$. Em trabalho desenvolvido por Hunsche et al. (2007), os ditiocarbamatos foram determinados por sua porção metálica (manganês).

Para remoção dos resíduos de agrotóxicos, alguns tratamentos têm sido estudados, dentre eles soluções ácidas, soluções básicas, água ozonizada, nanofiltração, condições climáticas, combinação de oxidação e tratamento biológico, métodos enzimáticos e zeólitas (Gonzalez et al. 1988, Fay et al. 2004, Lemic et al. 2006, Wu et al. 2007).

Os etileno-bis-ditiocarbamatos (exemplo: mancozeb), quando decompostos no ambiente ou biotransformados, produzem etilenotiureia (ETU), composto teratogênico, mutagênico e carcinogênico, provocando hiperplasia da tireóide e alterações significativas aos níveis séricos dos hormônios tireoidianos, no homem e em animais de laboratório. Existem evidências de que ETU pode, também, ser formada durante o processamento de alimentos contaminados (Oga 1996, Midio \& Martins 2000).

O objetivo deste trabalho foi avaliar a diminuição da concentração de mancozeb em tomates de mesa, com e sem remoção da película do fruto, utilizando-se processos de enxágue com água, solução com vinagre de álcool, solução com dicloroisocianurato de sódio dihidratado e solução com bicarbonato de sódio.

\section{MATERIAL E MÉTODOS}

Foram utilizados frutos de planta de tomateiro (Lycopersicum esculentum Mill.), cultivar Santa Clara, cultivados em casa de vegetação Poly Venlo (Van der Hoeven), revestida de policarbonato, com controle de temperatura. $\mathrm{O}$ transplante das mudas foi realizado em outubro de 2007, em 36 vasos de 5,0 L, contendo mistura de solo, esterco e areia, na proporção 3:1:1 $(\mathrm{v} / \mathrm{v})$. As plantas foram conduzidas em haste única e tutoradas com hastes de bambu. A adubação de base consistiu de $50 \mathrm{~g}$ da formulação N-P-K 4-14-8, adicionada ao substrato em cada vaso. A cada 20 dias, as plantas receberam $50 \mathrm{~mL}$ de adubo foliar Ouro Verde (Fertilizantes Ouro Verde), diluído em água ( $2 \mathrm{~g} / \mathrm{L}$ ) e aplicado diretamente ao substrato. A irrigação foi realizada com mangueira direcionada ao substrato. Empregaram-se outras práticas culturais adequadas para o correto manejo da cultura, incluindo o manejo de pragas e doenças, de acordo com Filgueira (2008). As colheitas iniciaram-se em 20 de dezembro de 2007 e estenderam-se até 15 de fevereiro de 2008.

Antes de se iniciarem os tratamentos com fungicidas, as plantas foram retiradas da estufa e mantidas ao ar livre, por 48 horas, até o momento da aplicação dos produtos. O fungicida mancozeb (produto comercial Manzate 800) foi pulverizado nos tomateiros, na dose de $3,0 \mathrm{~kg} \mathrm{ha}^{-1}$ do produto comercial, recomendada pelo fabricante (Du Pont do Brasil S. A. 2005), o que corresponde a $2,4 \mathrm{~kg} \mathrm{ha}^{-1}$ do ingrediente ativo. Foi aplicado volume correspondente a $400 \mathrm{~L} \mathrm{ha}^{-1}$, utilizando-se pulverizador costal com bico tipo cônico, com pressão aproximada de $200 \mathrm{lb} \mathrm{pol}^{-2}$, procedendo-se a uma cobertura uniforme, até o ponto de escorrimento. O fungicida foi aplicado a 45-50 $\mathrm{cm}$ acima da folhagem (Hunsche et al. 2007). Os frutos foram coletados após 24 horas da aplicação, para maior aderência do fungicida à superfície. Para representar o controle (frutos sem mancozeb), tomates, no ponto de colheita "verde-amarelado", foram coletados, antes da aplicação do fungicida.

Foram colhidos, aproximadamente, $5 \mathrm{~kg}$ de tomate, os quais foram imediatamente transportados ao Laboratório de Embalagem (DTA/UFV) e acondicionados em sacos plásticos de polietileno de baixa densidade. As amostras foram separadas em cinco lotes e submetidas aos diferentes tratamentos de enxágue por imersão (10 minutos, a $\left.27 \pm 1^{\circ} \mathrm{C}\right)$ : 1) água potável; 2) solução de vinagre de álcool a $10 \%$; 3 ) solução de dicloroisocianurato de sódio dihidratado a $0,66 \%$; e 
4) bicarbonato de sódio a $10 \%$. O lote denominado controle não foi submetido a enxágues.

\section{Análises físicoquímicas}

Antes da pulverização do fungicida, amostras de tomates foram submetidas a análises de $\mathrm{pH}$, em pHmetro Digimed (modelo DM 20); acidez titulável e teor de sólidos solúveis, segundo metodologia desenvolvida pelo Instituto Adolfo Lutz (2005); e atividade de água ( $\mathrm{Aw}$ ), determinada em equipamento Texto (modelo AG 650).

\section{Quantificação do Fungicida}

Para quantificação do fungicida, foi realizada análise do teor de manganês no tomate, antes da pulverização do mancozeb e após os tratamentos de enxague. As análises foram realizadas no Laboratório de Nutrição e Solos Florestais do Departamento de Solos da UFV (2008), segundo metodologia descrita por Fernandes et al. (2002), com adaptações das condições de secagem dos frutos a $65^{\circ} \mathrm{C}$, por 96 horas. Utilizou-se espectrômetro de Absorção Atômica, em equipamento Varian SpectraAA (modelo 2020FS), sendo a concentração de manganês expressa em mg kg-1 de tomate, em base seca (b.s.). Para o cálculo das concentrações de resíduos de mancozeb, foi obtida a curva padrão, utilizando-se soluções de manganês (Merck KGaA, Darmstadt e Germany), nas concentrações de $2 \mathrm{mg} \mathrm{L}^{-1}, 4 \mathrm{mg} \mathrm{L}^{-1}, 6 \mathrm{mg} \mathrm{L}^{-1}$, $8 \mathrm{mg} \mathrm{L}^{-1}$ e $10 \mathrm{mg} \mathrm{L}^{-1}$. Os resultados foram expressos em mg kg-1, multiplicando-se os dados obtidos pelo fator de conversão 4,939.

A concentração de mancozeb foi determinada na película e no fruto inteiro, em cada tratamento. Para a análise da película, os frutos foram descascados, manualmente, a uma profundidade de, aproximadamente, $1 \mathrm{~mm}$.

Para avaliar a eficiência do procedimento analítico de recuperação do mancozeb, amostras de frutos isentos do produto foram contaminadas pela adição do fungicida, de forma a conter concentrações de $0,4 \mathrm{mg} \mathrm{g}^{-1} ; 1,2 \mathrm{mg} \mathrm{g}^{-1}$; e $2,4 \mathrm{mg} \mathrm{g}^{-1}$, e, posteriormente, foi determinado o teor de manganês. Os resultados foram expressos em porcentagem (\%) e calculados conforme a seguinte equação:

Eficiência (\%) $=\frac{\text { mancozeb recuperado }\left(\mathrm{mg} \mathrm{g}^{-1}\right)}{\text { mancozeb adicionado }\left(\mathrm{mg} \mathrm{g}^{-1}\right)} \times 100$

\section{Análise estatística}

O experimento foi conduzido em delineamento inteiramente casualizado, no esquema de parcelas subdivididas, sendo as parcelas compostas pelas quatro soluções de enxágue (água potável, solução de vinagre de álcool, solução de bicarbonato de sódio e solução de dicloroisocianurato de sódio dihidratado) e as subparcelas constituídas pelas partes avaliadas dos frutos (frutos com e sem remoção da película). Foram realizadas duas repetições e cada análise foi desenvolvida em triplicata. Os dados obtidos foram submetidos a análise de variância (Teste F) e as médias dos níveis de cada fator comparadas entre si, pelo teste Tukey, a $5 \%$ de probabilidade, utilizando-se o programa Statistica, versão 6.0 (StatSoft).

\section{RESULTADOS E DISCUSSÃO}

Os valores de $\mathrm{pH}$, sólidos solúveis e acidez titulável (Tabela 1) aproximaram-se dos encontrados por Chiumarelli \& Ferreira (2004), que foram de 4,2; $4,4^{\circ}$ Brix; e $0,24 \mathrm{~g}$ de ácido cítrico por $100 \mathrm{~g}$ de polpa, respectivamente, para a cultivar Débora. Outros estudos com tomate revelam valores de $\mathrm{pH}$ entre 4,0 e 4,5 (Embrapa 2000, Fernandes et al. 2002). A importância do pH, citada por Borguini (2002), foi a sua relação com a acidez na aceitação do tomate. O teor de sólidos solúveis $\left(5,0^{\circ}\right.$ Brix $)$ foi semelhante ao encontrado por Fernandes et al. (2002), em tomate Santa Clara. Segundo Chitarra \& Chitarra (1990), o teor de sólidos solúveis totais avalia o grau de doçura do fruto. A acidez titulável das amostras foi de

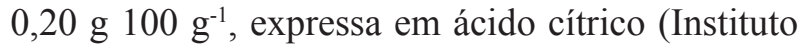
Adolfo Lutz 2005). Segundo Chitarra \& Chitarra (1990), os ácidos orgânicos estão dissolvidos nos vacúolos das células, tanto na forma livre como combinada com sais, ésteres e glicosídeos. Esses ácidos orgânicos contribuem, assim, para a acidez do fruto.

Tabela 1. Características físicoquímicas de tomate, cultivar Santa Clara, cultivado sem aplicação de mancozeb (Viçosa, MG, 2008).

\begin{tabular}{lc}
\hline \multicolumn{1}{c}{ Análises físicoquímicas } & Média \pm Desvio Padrão \\
\hline Acidez (mg de ácido cítrico $\left.100 \mathrm{~g}^{-1}\right)$ & $0,20 \pm 0,001$ \\
$\mathrm{pH}$ & $4,60 \pm 0,060$ \\
Sólidos Solúveis ( ${ }^{\circ}$ Brix) & $5,00 \pm 0,001$ \\
Relação Brix/acidez & $25,00 \pm 0,001$ \\
Aw & $0,96 \pm 0,010$ \\
Teor de manganês $\left(\mathrm{mg} 100 \mathrm{~g}^{-1}\right.$ (b.s.)) & $1,56 \pm 0,020$ \\
\hline
\end{tabular}


Borguini (2002) encontrou valores na faixa de $0,33 \mathrm{~g}$

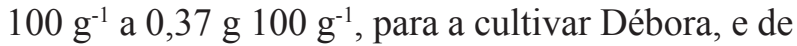

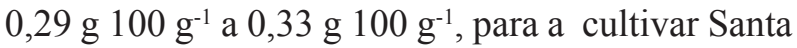
Clara. Fernandes et al. (2002) encontraram valor

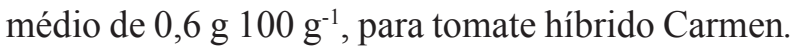
A relação Brix/acidez foi 25, indicando elevado grau de maturação. Chiumarelli \& Ferreira (2004) encontraram valor médio de 18,3, para tomates Débora.

A atividade de água (Aw) média encontrada para os tomates foi de 0,96 (Tabela 1). Segundo Christensen \& Kaufmann (1974), a Aw é a disponibilidade de água para atuar como solvente e participar das reações químicas, bioquímicas e microbiológicas. De acordo com Azeredo (2004), na maior parte dos alimentos frescos, a Aw é superior a 0,95.

$\mathrm{O}$ teor de manganês $(\mathrm{Mn})$ encontrado na amostra de tomate sem aplicação de fungicida foi de $1,56 \mathrm{mg} 100 \mathrm{~g}^{-1} \pm 0,02$. Em estudo realizado por Borguini (2002), os valores oscilaram entre $0,06 \mathrm{mg} 100 \mathrm{~g}^{-1}$ e $0,17 \mathrm{mg} 100 \mathrm{~g}^{-1}$ no fruto, valores muito abaixo dos observados neste estudo. Contudo, Fernandes et al. (2002) encontraram, em tomates hi-

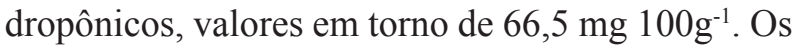
autores mencionam que as altas concentrações podem ser justificadas pela contaminação durante o processo de adubação. Lima \& Oliveira (2003) avaliaram a variabilidade espacial de nutrientes, entre eles o manganês, nos diversos tipos de solos, encontrando coeficiente de variação de até $204,1 \%$.

A eficiência do processo de recuperação do mancozeb, nas concentrações $0,4 \mathrm{mg} \mathrm{g}^{-1} ; 1,2 \mathrm{mg} \mathrm{g}^{-1}$; e $2,4 \mathrm{mg} \cdot \mathrm{g}^{-1}$, foram de $103 \pm 7,3 \% ; 72,5 \pm 8,9 \%$; e $67,3 \pm 19,7 \%$, respectivamente. A eficiência de quantificação da recuperação encontrada, para análises de agrotóxicos, é de $70 \%$ a $120 \%$, conforme Garp (1999). Esta faixa de recuperação é admitida devido à complexidade das matrizes analisadas, uma vez que vários elementos constituintes do fruto podem interferir no resultado final da análise (Vieira \& Lichtig 2004).

Pelos resultados obtidos (Figura 1), verificouse que, tanto na avaliação da película de tomate quanto no tomate inteiro, as soluções de enxágue promoveram reduções significativas na concentração do fungicida, se comparadas à testemunha $(\mathrm{p} \leq 0,05)$, não sendo observada interação entre os fatores. Entretanto, as médias de concentração do mancozeb não diferiram entre as quatro soluções testadas.

Antes do enxágue, a concentração de mancozeb foi de 593,52 $\mathrm{mg} \mathrm{kg}^{-1}$, na película, e de

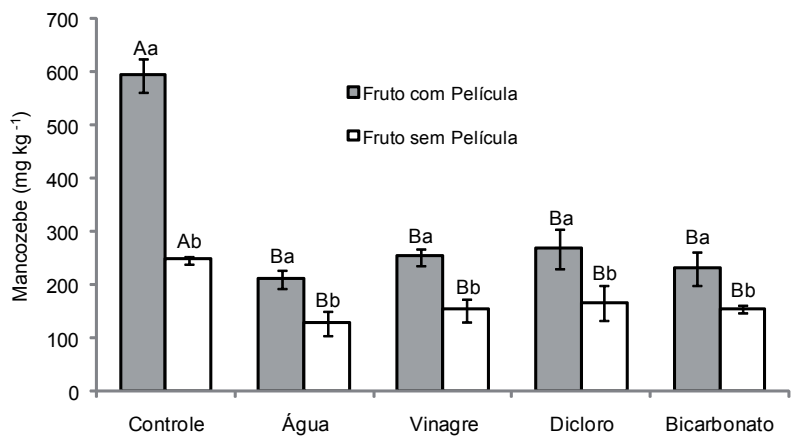

Figura 1. Concentração de resíduos de mancozeb em tomate (película e fruto inteiro, após imersão). Letras maiúsculas $=$ enxágues e letras minúsculas $=$ película $\mathrm{x}$ tomate inteiro. Colunas (tratamentos) com a mesma letra não diferem, significativamente $(\mathrm{p}>0,05)$, pelo teste Tukey. Tratamentos: controle (sem enxágue); água potável; vinagre de álcool $10 \%$; dicloro = dicloroisocianurato de sódio dihidratado $3 \%$; e bicarbonato de sódio $10 \%$, por 10 minutos.

$247,89 \mathrm{mg} \mathrm{kg}^{-1}$, no fruto inteiro. A maior concentração de mancozeb na película deve-se, provavelmente, ao caráter não sistêmico do produto. Zohair (2001) realizou pesquisa com agrotóxicos não sistêmicos, em batatas (película e polpa), encontrando níveis de organofosforados em torno de $41,50 \mu \mathrm{g} \cdot \mathrm{kg}^{-1}$, na película, e ausência, na polpa. Após enxágue, a concentração de mancozeb foi reduzida para valores entre $211,68 \mathrm{mg} \mathrm{kg}^{-1}$ (enxágue com água) e 267,91 $\mathrm{mg} \mathrm{kg}^{-1}$ (enxágue com dicloro), na película, e $128,13 \mathrm{mg} \mathrm{kg}^{-1}$ (enxágue com água) e $167,07 \mathrm{mg} \mathrm{kg}^{-1}$ (enxágue com dicloro), no fruto inteiro.

A redução do mancozeb, neste estudo, variou de $50,2 \%$ a $62,1 \%$, na película, e de $32,8 \%$ a $48,4 \%$, no fruto inteiro (Tabela 2). A maior remoção observada na película, em relação ao fruto inteiro, está, provavelmente, relacionada à distribuição do fungicida, que apresenta maior concentração na su-

Tabela 2. Porcentagem de redução de resíduos de mancozeb, após enxágue de tomates in natura (cultivar Santa Clara).

\begin{tabular}{|c|c|c|}
\hline Tratamentos & $\begin{array}{c}\text { Película } \\
\left(\text { Média } \pm \mathrm{DP}^{1}\right)\end{array}$ & $\begin{array}{l}\text { Fruto inteiro } \\
\left(\text { Média } \pm \mathrm{DP}^{1}\right)\end{array}$ \\
\hline Água potável & $62,1 \pm 17,9$ & $48,4 \pm 22,8$ \\
\hline Vinagre de álcool $10 \%$ & $57,3 \pm 16,2$ & $38,1 \pm 21,2$ \\
\hline $\begin{array}{l}\text { Dicloroisocianurato } \\
\text { de sódio dehidratado 3\% }\end{array}$ & $50,2 \pm 36,9$ & $32,8 \pm 33,6$ \\
\hline Bicarbonato de Sódio 10\% & $61,2 \pm 31,5$ & $37,3 \pm 8,2$ \\
\hline
\end{tabular}

'Desvio padrão. 
perfície do tomate, e aos diferentes fatores que afetam a sua degradação. Segundo Gleber et al. (2006), a degradação dos agrotóxicos e a consequente remoção estão relacionadas aos processos químicos, físicos e biológicos, como fotólise, oxidação, adsorção, volatilização e hidrólise, entre outros.

Hwang et al. (2002) obtiveram redução parcial do mancozeb em maçãs, utilizando enxágue em água de torneira (48,1\%), ácido peracético a 50 ppm $(82,1 \%)$ e dióxido de cloro a 10 ppm $(87,9 \%)$, e redução total com hipoclorito de cálcio a 500 ppm e água ozonizada a 3 ppm.

\section{CONCLUSÕES}

1. A maior quantidade de mancozeb está presente na película do produto e sua remoção poderia reduzir, aproximadamente, até $70 \%$ do fungicida.

2. A imersão dos frutos em água potável, por 10 minutos, aliada à retirada da película, poderá ser suficiente para reduzir a concentração do mancozeb, no produto final, a níveis toleráveis.

\section{AGRADECIMENTOS}

Aos pesquisadores dos Laboratórios de Embalagem do Departamento de Tecnologia de Alimentos e dos Laboratórios de Solos e Florestais do Departamento de Solos, da Universidade Federal de Viçosa, pela contribuição no desenvolvimento do estudo, e à Capes, pelo apoio financeiro.

\section{REFERÊNCIAS}

ARBO, M. D. et al. Efeito tóxico dos praguicidas maneb e paraquat sobre a atividade da enzima antioxidante catalase em ratos. Revista de Ciências Farmacêuticas Básica e Aplicada, Porto Alegre, v. 27, n. 1, p. 57-61, 2006.

ASSOCIAÇÃO GRUPO DE ANALISTAS DE RESÍDUOS DE PESTICIDAS (GARP). Manual de resíduos de pesticidas em alimentos. São Paulo: GARP, 1999.

AZEREDO, H. M. C. Fundamentos de estabilidade de alimentos. Fortaleza: MAPA/ Embrapa Agroindústria Tropical, 2004.

BASTOS, L. H. P. et al. Ensaio de proficiência para análise de ditiocarbamatos em polpa de banana. Revista Química Nova, Rio de Janeiro, v. 30, n. 1, p. 32-35, 2007.
BEDOR, C. et al. Avaliação e reflexos da comercialização e utilização de agrotóxicos na região do submédio do Vale do São Francisco. Revista de Saúde Pública, Petrolina, v. 31, n. 1, p. 68-76, 2007.

BORGUINI, R. G. Tomate (Lycopersicum esculentum Mill.) orgânico: o conteúdo nutricional e a opinião do consumidor. 2002. 110 f. Dissertação (Mestrado em Agronomia)-Universidade de São Paulo, Escola Superior de Agricultura Luiz de Queiroz, Piracicaba, 2002.

CHITARRA, M. I. F; CHITARRA, A. B. Pós-colheita de frutos e hortaliças: fisiologia e manuseio. Lavras: ESAL/ FAEPE, 1990.

CHIUMARELLI, M.; FERREIRA, M. D. Avaliação da qualidade pós-colheita de tomate de mesa com utilização de coberturas comestíveis a $25^{\circ} \mathrm{C}$. In: CONGRESSO BRASILEIRO DE ENGENHARIA AGRÍCOLA, 23., 2004, São Pedro. Anais... Campinas: Unicamp, 2004. p. 1-4.

CHRISTENSEN, C. M.; KAUFMANN, H. H. Microflora. In: CHRISTENSEN, C. M. Storage of cereal grain and their products. St. Paul: American Association of Cereal Chemists, 1974. p. 158-192.

DEBBARH, I. et al. Human neurotoxicity of ethylenebis-dithiocarbamates (EBDC). Revue Neurologique, Paris, v. 158, n. 12, p. 1175-1180, 2002.

DELGAdo, I. F.; PAUMGARTten, F. J. R. Intoxicações e uso de pesticidas por agricultores do município de Paty do Alferes, Rio de Janeiro, Brasil. Caderno de Saúde Pública, Rio de Janeiro, v. 20, n. 1, p.180-186, 2004.

DU PONT DO BRASIL S.A. Especificações do produto Manzate 800. Barueri: Du Pont do Brasil, 2005.

EMPRESA BRASILEIRA DE PESQUISA AGROPECUÁRIA (Embrapa Hortaliças). Tabela de composição nutricional das hortaliças. 2000. Disponível em: <http://www.cnph.embrapa.br/util/tabelahortalicas. htm\#inicio>. Acesso em: 31 jan. 2008.

FAY, E. F. et al. Remoção de resíduos de ditiocarbamatos e de etilenotioureia. In: SEMINÁRIO BRASILEIRO DE PRODUÇÃO INTEGRADA DE FRUTAS, 5., 2004, Petrolina. Anais... Petrolina: Embrapa/CPATSA, 2004. p. 1-17.

FERNANDES, A. A.; MARTINEZ, H. E. P.; FONTES, P. C. R. Produtividade, qualidade dos frutos e estado nutricional do tomateiro tipo longa vida conduzido com um cacho, em cultivo hidropônico, em função das fontes de nutrientes. Horticultura Brasileira, Brasília, DF, v. 20, n.4, p. 564-570, 2002. 
FILGUEIRA, F. A. R. Novo manual de olericultura. 3. ed. Viçosa: UFV, 2008.

GLEBER, L.; PELIZZA, T. R.; AlMEIDA, D. L. Variáveis ambientais e toxicológicas de agroquímicos utilizados na produção integrada de maçãs (PIM) visando modelagem matemática. Revista de Ciências Agroveterinárias, Lages, v. 5, n. 2, p.169-184, 2006.

GONZALEZ, A. R. et al. Effect of leaf type, leaf injury, storage temperature and washing solution on ethylenethiourea residues of carbamate-treated canned spinach. Journal of Food Safety, Washington, v. 9 , n. 3, p. 155-164, 1988.

HUNSCHE, M. et al. Mancozeb wash-off from apple seedlings by simulated rainfall as affected by drying time of fungicide deposit and rain characteristics. Crop Protection, Bonn, v. 26, n. 5, p. 768-774, 2007.

HWANG, E. S.; CASH, J. N.; ZABIK, M. J. Degradation of mancozeb and ethylenethiourea in apples due to postharvest treatments and processing. Food Chemistry and Toxicology, Ann Arbor, v. 67, n. 9, p. 3295-3300, 2002.

INSTITUTO ADOLFO LUTZ. Métodos físicoquímicos para análise de alimentos. 4. ed. Brasília, DF: Ministério da Saúde/Agência Nacional de Vigilância Sanitária, 2005.

JARDIM, A. N. O. Desafios analíticos para análise de agrotóxicos em alimentos. In: SIMPÓSIO BRASILEIRO DE RESÍDUOS DE AGROTÓXICOS EM ALIMENTOS, 2., 2007, Búzios. Disponível em: <http//www.anvisa.gov.br/divulga/eventos/ Simpósio res agrotoxicos/simpósio- $221107 /$ ii_simposio_residuos.prog.pdf $>$. Acesso em: 14 jan. $2 \overline{0} 08$.

LEMES, V. R. et al. Avaliação de resíduos de ditiocarbamatos e etilenoureia (ETU) em mamão e sua aplicação na saúde pública. Revista do Instituto Adolfo Lutz, São Paulo, v. 64, n. 1, p. 50-57, 2005.

LEMIC, J. et al. Removal of atrazine, lindane and diazinone from water by organo-zeolites. Water Research, Belgrade, v. 40, n. 5, p. 1079-1085, 2006.
LIMA, C. B.; OLIVEIRA, M. Variabilidade espacial de cobre, ferro, manganês e zinco em solos da região oeste do Estado do Rio Grande do Norte. Caatinga, Mossoró, v. 16, n. 1, p. 63-67, 2003.

LIMA, R. S. et al. Biossensor enzimático para detecção de fungicidas ditiocarbamatos: estudo cinético da enzima aldeído desidrogenase e otimização do biossensor. Química Nova, São Luís, v. 30, n. 1, p. 9-17, 2007.

MIDIO, A. F.; MARTINS, D. I. Toxicologia de alimentos. São Paulo: Varela, 2000.

OGA, S. Fundamentos de toxicologia. São Paulo: Atheneu, 1996.

VETORAZZI, G. et al. International safety assessment of pesticides: dithiocarbamate pesticide, ETU, and PTU - a review and update. Teratogenesis, Carcinogenesis, and Mutagenesis, New York, v. 15, n. 6, p. 313-317, 1995.

VIEIRA, E.; LICHTIG, J. Validação de métodos cromatográficos em análises de resíduos de pesticidas. Arquivos do Instituto Biológico, São Paulo, v. 71, supl., p. 1-749, 2004.

WU, J. G. et al. Efficacy evaluation of lowconcentration of ozonated water in removal of residual diazinon, parathion, methyl-parathion and cypermethrin on vegetable. Journal of Food Engineering, Hong Kong, v. 79, n. 3, p. 803-809, 2007.

ZHOU, Y. et al. Proteasomal inhibition induced by manganese ethylenebis-dithiocarbamate: relevance to Parkinson's disease. Neuroscience, Oxford, v. 128, n. 2, p. 281-291, 2004.

ZOHAIR, A. Behaviour of some organophosphorus and organochlorine pesticides in potatoes during soaking in different solutions. Food and Chemical Toxicology, Ashmoun, v. 39, n. 7, p. 751-755, 2001. 